\title{
Chronic myeloid leukemia (CML): prognostic factors and survival analysis
}

\author{
Disciplina de Hematologia e Hemoterapia, Escola Paulista de Medicina,
} Universidade Federal de São Paulo - São Paulo, Brazil

\begin{abstract}
The prognostic value of different factors upon diagnosis of CML was analysed in 45 Philadelphia (Ph1)-positive patients. The median survival was 48 months. Univariate analysis showed 5 poor prognostic factors (male sex, under 45 years-old, bone marrow blasts greater than or equal to 10 percent, blood basophils greater than or equal to 6 percent and blood eosinophils greater than or equal to 6 percent) which provided for the development of a clinical staging system: Stage I with none or one factor and a two-year survival rate of 100 percent; Stage II with two or three factors and two-year survival of 72.2 percent; and Stage III with four or five factors and twoyear survival of 0 percent $(p=0.00016)$. Multivariate survival analysis showed that combination of blood basophilia and bone marrow blasts had the strongest predictive relationship to survival time. We conclude that a combination of pretreatment factors identifies different risk subcategories in CML patients and is helpful in assessing the overall prognosis and the treatment approach.
\end{abstract}

UNITERMS: Chronic Myeloid Leukemia. Philadelphia Chromosome. Prognosis.

\section{INTRODUCTION}

$\mathrm{T}$ The analysis of prognostic factors has allowed for the elaboration of staging systems in order to plan for the treatment ${ }^{2.17}$ of several hematologic diseases. In CML, these studies have been used to guide the best therapeutic approach in each case, such as bone marrow transplantation, which should be implemented as soon as possible during the chronic phase in young patients with a compatible donor.

\section{OBJECTIVE}

This study seeks to analyze the prognostic significance of different features detected at diagnosis in a series of CML patients from a public Brazilian hospital, in order to determine if the prognostic factors of this population were different from well-established factors detected in studies performed in other countries.

\section{MATERIAL AND METHODS}

We are reporting on the analysis of 45 adult $\mathrm{Ph} 1$ positive CML patients in the chronic phase who were admitted to our hospital between September 1977 and 
January 1993. The follow-up period varied from 1 to 184 months. There were 7 ( 15.5 percent) patients with a follow-up of less than 1 year, and 3 (6.7 percent) with less than 6 months. The diagnosis of CML was based on conventional criteria. The karyotype was evaluated by usual methods with $G$ banding and classified according to $\operatorname{ISCN}(1985,1991)$. The characterization of blast crisis was made from the analysis of bone marrow aspiration (more than 30 percent of blasts), and the type of crisis was determined by cytochemistry and immunophenotyping methods. Patients in the chronic or accelerated phase were treated with busulfan or hydroxyurea. Lymphoid blast crisis was treated with vincristine, daunorubicin and prednisone, and myeloid blast crisis with daunorubicin and cytosine arabinoside, until patients returned to the chronic phase when busulfan or hydroxyurea was restarted. At diagnosis, the following clinical and hematological data were recorded and evaluated for prognosis: 1) age, sex, race, spleen and liver size; 2) peripheral blood features: hemoglobin concentration $(\mathrm{Hb})$, platelet counts, white blood cell counts (WBC) with differential counts; and blood erythroblast percentage; 3 ) bone marrow aspiration features: blast cells percentage and myeloid/ erythroid $(\mathrm{M} / \mathrm{E})$ ratio; 4 ) bone marrow biopsy features: granulocytic proliferation (Gran) or granulocytic plus megakaryocytic proliferation (Gran/Meg), eosinophilia, presence of blast cells, fibrosis and megakaryocyte morphology ${ }^{10}$. For patients who discontinued followup sometime during this study, the survival period was defined as the date when the patient was last seen; for patients who died, the survival period was defined as the date of death; for patients who were alive in May 1993, the survival period was considered to be until that date.

For univariate analysis, the cut-off level of each quantitative variable was established based on those commonly found in the literature data. In some cases, this was established by chance, until "p" values near 5 percent were found. The different categories of qualitative variables, such as sex, race and bone marrow histology, were compared to each other. Actuarial survival probability curves were plotted according to
Kaplan and Meyer's method ${ }^{12}$. Different curves were statistically compared using the Cox-Mantel (log rank) or the generalized Wilcoxon Test ${ }^{13}$. The staging system was derived from univariate analysis using the variables associated with bad prognosis. Thus, the low-risk or Stage I group consisted of patients with zero or one factor; the intermediate-risk group or Stage II consisted of 2 or 3 factors and the high-risk group, 4 or 5 factors. Using the Cox model $^{4}$, we performed a multivariate analysis of all variables previously selected by univariate analysis, which were then put into a single equation to determine the variables which were primarily important to prognosis and those which were only secondary factors.

\section{RESULTS}

There were 25 (55.6 percent) males and 20 (44.4 percent) females. The median age was 42 (ranging from 15-77). There were 25 (55.6 percent) white patients, 18 (40.0 percent) were black, and 2 (4.4 percent) were Asian. The median survival was 48 months (Fig. 1). At the time the analysis was conducted in May 1993, 15 (33.4 percent) patients had died, 23 (51.1 percent) were alive and 7 (15.5 percent) had discontinued follow-up some time after diagnosis. Of the 23 living patients, 18 ( 78.3 percent) were in the chronic phase, 3 (13.0 percent) in the accelerated phase, and 2 ( 8.7 percent) were alive after blastic phase.

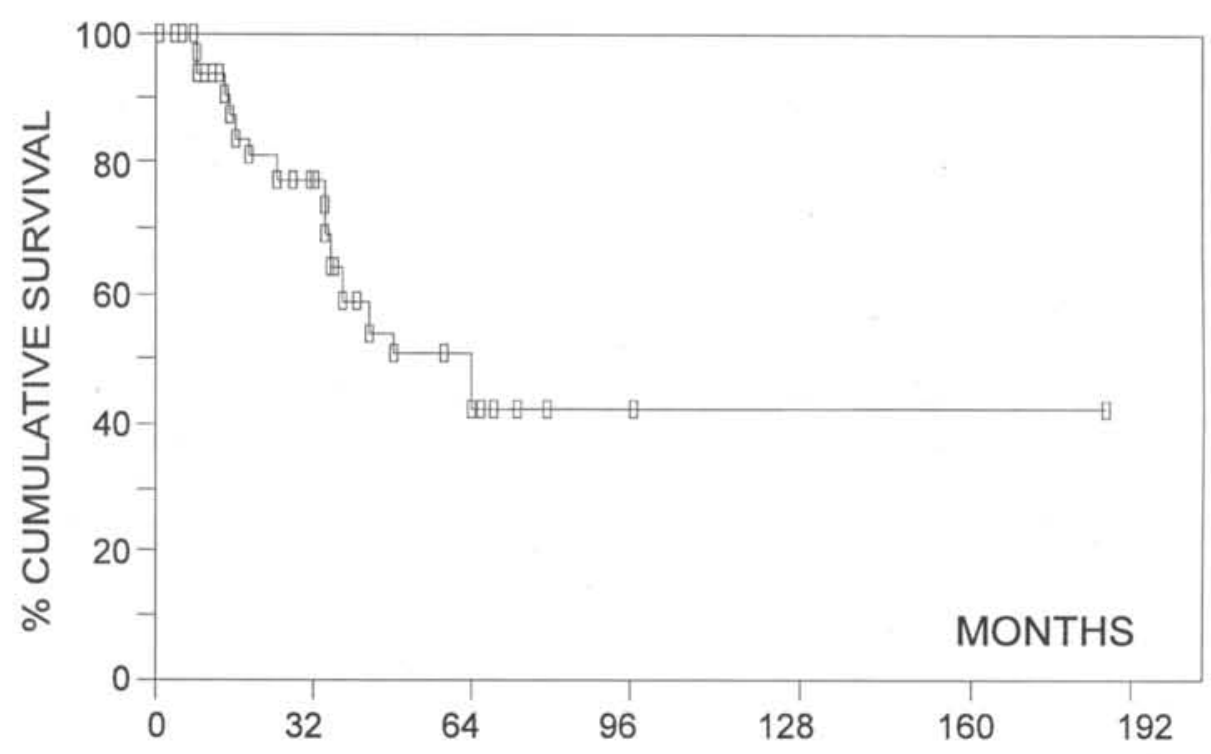

Figure 1 - General survival of $45 \mathrm{CML}$ Ph1-positive patients. 
Table 1

Results of the univariate analysis

\begin{tabular}{|c|c|c|c|c|c|}
\hline Factor & & $\mathrm{N}$ & & $\mathrm{p}$ & \\
\hline SEX & mal & & & 25 & $0.04664^{*}$ \\
\hline & fem & & & 20 & \\
\hline AGE & $<45$ & & & 24 & $0.00783^{*}$ \\
\hline & $>=4$ & & & 21 & \\
\hline & $<60$ & & & 33 & 0.26745 \\
\hline & $>=6$ & & & 12 & \\
\hline RACE & whit & & & 25 & 0.73522 \\
\hline & blac & & & 18 & \\
\hline SPLEE & No-1 & $\mathrm{cm}$ & & 19 & 0.07330 \\
\hline & & $>10 \mathrm{~cm}$ & & 23 & \\
\hline LIVER & nor & & & 17 & 0.52875 \\
\hline & enta & arged & & 26 & \\
\hline $\mathrm{Hb}$ (FEN & AALE & $<12 \mathrm{~g} \%$ & & 15 & 0.63783 \\
\hline & & $>=12 \mathrm{~g} \%$ & & 04 & \\
\hline $\mathrm{Hb}(\mathrm{MA}$ & & $<13 \mathrm{~g} \%$ & & 23 & \\
\hline & & $>=13 \mathrm{~g} \%$ & & 00 & \\
\hline WBC & $<1$ & $00 \times 109 / 1$ & & 16 & 0.39870 \\
\hline & $>=1$ & $00 \times 109 / 1$ & & 26 & \\
\hline PLATE & -ET & $<400 \times 10^{9} / 1$ & & 27 & 0.15681 \\
\hline & & $>=400 \times 10^{9} /$ & & 14 & \\
\hline & & $150<x<500\rangle$ & $10^{9} /$ & 11 & 0.88121 \\
\hline & & $<150$ or $>50$ & & 30 & \\
\hline ERYTH & ROB & LASTS & $\%$ & 34 & 0.39574 \\
\hline & & & $\%$ & 11 & \\
\hline PERIPI & IERA & L BLASTS & $0-1$ & 22 & 0.56602 \\
\hline & & & $>1$ & 20 & \\
\hline & & & $<5 \%$ & 35 & 0.77335 \\
\hline & & & $>=5 \%$ & 07 & \\
\hline & & & $<10 \%$ & 40 & 0.27416 \\
\hline & & & $>=10 \%$ & 02 & \\
\hline BM BL. & ASTS & $<10 \%$ & & 38 & $0.00121^{\circ}$ \\
\hline & & $>=10 \%$ & & 02 & \\
\hline BLOOD & BAS & SOPHILS & $<6 \%$ & 32 & $0.00010^{\circ}$ \\
\hline & & & $>=6 \%$ & 12 & \\
\hline BLOOL & EOS & IINOPHILS & $<6 \%$ & 32 & $0.02938^{\circ}$ \\
\hline & & & $>=6 \%$ & 10 & \\
\hline$M / E R A$ & TIO & $<20$ & & 17 & 0.70445 \\
\hline & & $>=20$ & & 15 & \\
\hline & & $<30$ & & 22 & 0.64397 \\
\hline & & $>=30$ & & 10 & \\
\hline BM & GR & AN & & 12 & 0.74759 \\
\hline & & AN/MEG & & 16 & \\
\hline FIBRO & & $0 /+$ & & 09 & 0.42116 \\
\hline & & $++/+++$ & & 19 & \\
\hline
\end{tabular}

OBS: $\mathrm{N}=$ number of patients; ${ }^{-}=\mathrm{p} 0.05 ; \mathrm{Hb}=$ hemoglobin; $\mathrm{BM}=$ bone marrow; $M / E$ = myeloid/erythroid
Amongst all the patients, the number of blast crises was 16 , with 2 (12.5 percent) patients still alive and 14 (87.5 percent) dead. There were 6 ( 37.5 percent) lymphoid blast crises, 9 (56.3 percent) myeloid blast crises and 1 (6.2 percent) unclassified. The mean survival of all blast crises was 4.5 months, with 6.2 months for lymphoid and 4.1 months for myeloid, but this difference was not significant $(\mathrm{p}=0.22285)$. Death was due to blastic transformation in 14 (93.3 percent) cases, and in 1 patient ( 6.7 percent), to other complications. The univariate analysis demonstrated 5 variables that seemed to be associated with poor prognosis $(\mathrm{p}<0.05)$ : male sex $(\mathrm{p}=0.04664)$, aged $<45$ $(\mathrm{p}=0.00783)$; bone marrow blasts greater than or equal to 10 percent $(\mathrm{p}=0.00121)$; blood basophils greater than or equal to 6 percent ( $\mathrm{p}=0.00010)$; and blood eosinophils greater than or equal to 6 percent $(\mathrm{p}=0.02938)$. The other variables, such as spleen size, liver size, hemoglobin level, white blood cell counts, platelet counts, peripheral blasts, circulating erythroblasts, $\mathrm{M} / \mathrm{E}$ ratio and bone marrow features (Gran or Gran/Meg and fibrosis), were not statistically significant at the different cut-off levels tested (Table 1).

The staging system, derived from the univariate analysis, permitted the population to be divided into 3 groups: Stage I with 19 (42.2 percent) patients, Stage II with 16 (35.5 percent) and Stage III with 4 (8.8 percent). Six (13.3 percent) patients were excluded due to lack of data on one or more selected variables. The death rate was 0.0 percent $(0 / 19)$ for Stage I, 37.5 percent $(6 / 16)$ for Stage II, and 75.5 percent (3/4) for Stage III, and this difference was significant $(\mathrm{p}=0.00016)$ (Fig. 2). Multivariate analysis was performed on 38 patients who had data for 5 variables selected by univariate analysis and 4 variables of prognostic importance (hemoglobin, spleen, platelet counts and peripheral blast cells). Only two variables were identified as primarily important for prognosis: blood basophils $(\mathrm{p}=0.004)$ and bone marrow blasts $(p=0.042)$. The equation derived from multivariate analysis was:

$$
\begin{gathered}
\mathrm{RR}=\operatorname{EXP}\{0.2936(\mathrm{BM} B L-2.58)+0.2647 \\
(\mathrm{PB} \text { BASO }-4.21)\}
\end{gathered}
$$

$\mathrm{RR}=$ relative risk; $\mathrm{BM} \mathrm{BL}=\%$ bone marrow blasts; $\mathrm{PB} \mathrm{BASO}=$ $\%$ peripheral blood basophils; $2.58=$ mean of bone marrow blasts $(\%) ; 4.21=$ mean of blood basophils $(\%)$.

Using this analysis, patients were divided into 3 groups: low relative risk $(\mathrm{RR})(<1.0)$; intermediate $\mathrm{RR}$ (1.0 to 10.0); and high RR (>10.0. The Wilcoxon test showed that there was a significant difference of survival 


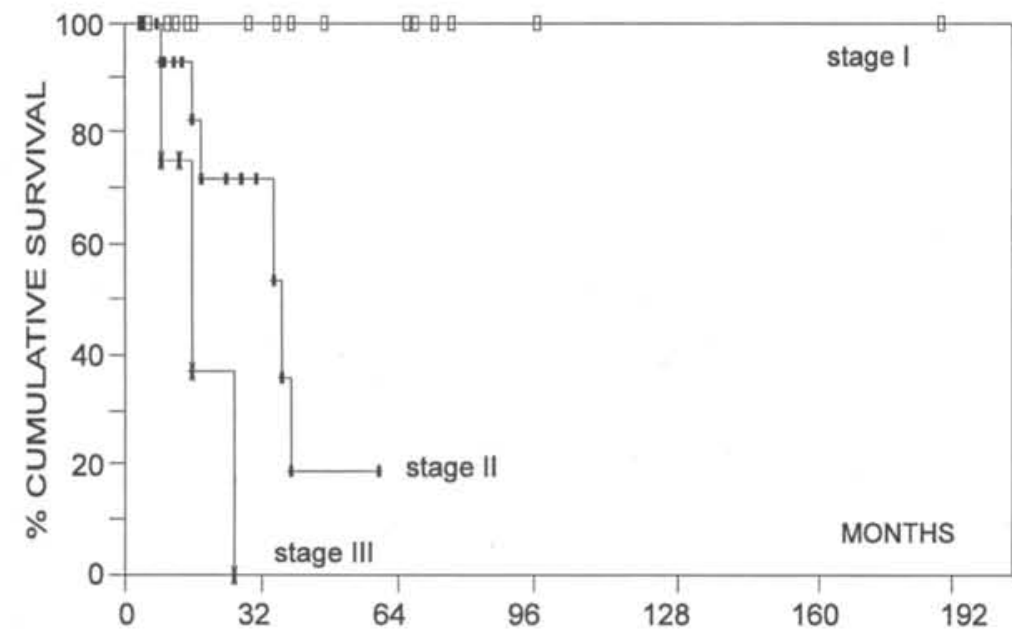

Figure 2 - Actuarial survival probability curves of Stages I, II and III, according to the proposed staging system $(p=0.00016)$.

in the 3 groups ( $\mathrm{p}=0.00000$ ) (Fig. 3 ). The actuarial death rate; 5.1 percent during the first year after dignosis, 18.1 percent during the second, 23.1 percent during the third, and 18.2 percent during the fourth, achieved a "plateau" after the fifth year.

\section{DISCUSSION}

The fatal outcome of CML has stimulated the analysis of prognostic factors. However, besides the absence of the $\mathrm{Ph} 1$ chromosome, which leads to a rapid evolution to the blastic phase $\mathrm{e}^{2.68 .10 .17 .18}$, there is no agreement as to the prognostic importance of the many different features at diagnosis.

The median age of 42 in this study was lower than expected ${ }^{15}$. The median survival of 48 months was in accordance with other authors ${ }^{2.8 .10 .16}$. There was no difference between survival and death rates after the onset of blast crises, although lymphoid blast crises seemed to be more responsive to conventional chemotherapy ${ }^{9}$. Male sex, as described by other authors ${ }^{10.17}$, and age under 45 were associated with an unfavorable prognosis ( $\mathrm{p}=0.04664$ and 0.00783 , respectively) in our study. In a worldwide multicentric study, SOKAL et al. ${ }^{18}$ described age as a factor with a progressively unfavorable prognosis at higher values.

Therefore, this is the first significant difference between our population and others previously studied. This aspect seems highly important, because patients under 45 yearsold are those referred for bone marrow transplantation. Unfortunately, the multivariate analysis did not confirm age as an important prognostic factor, perhaps due to the small number of patients in this study. As described by others ${ }^{2.10 .11}$, bone marrow blasts of 10 percent or more was another finding negatively related to survival in our population ( $\mathrm{p}=0.00121)$, which might be associated with an early accelerated phase. Marked peripheral basophilia at diagnosis was described as a poor prognostic factor $^{8.10 .11 .19}$, and was another significant negative factor in our study (6 percent or more $)(p=0.00010)$. Along the same line, eosinophilia which is or is not associated with basophilia has been related to a bad prognosis $\mathrm{s}^{8.17 .18}$, as shown in this report $(\mathrm{p}=0.02938)$. Other factors tested in this study such as spleen and liver size, white blood cell counts, platelet counts, peripheral blast cells, blood erythroblasts, $\mathrm{M} / \mathrm{E}$ ratio and bone marrow aspects (Gran or Gran/Meg and fibrosis) did not significantly affect the prognosis in our population. Some authors have described enlarged spleen ${ }^{2.10 .11 .18 .20}$ or liver $^{2.11 .20}$, low hemoglobin ${ }^{10.11}$, increased leukocyte counts $^{8,19}$, low or high platelet counts ${ }^{10.11 .20}$, high percentages of blasts in peripheral blood ${ }^{8.18 .20}$, presence of erythroblasts in peripheral blood ${ }^{2.17}$, high lactic dehidrogenase level ${ }^{8.17}$, high uric acid and an increased $\mathrm{M} / \mathrm{E}$ ratio ${ }^{8}$ as unfavorable factors at diagnosis. SOKAL et al. ${ }^{18}$ reported an actuarial death rate of $5-10$ percent in

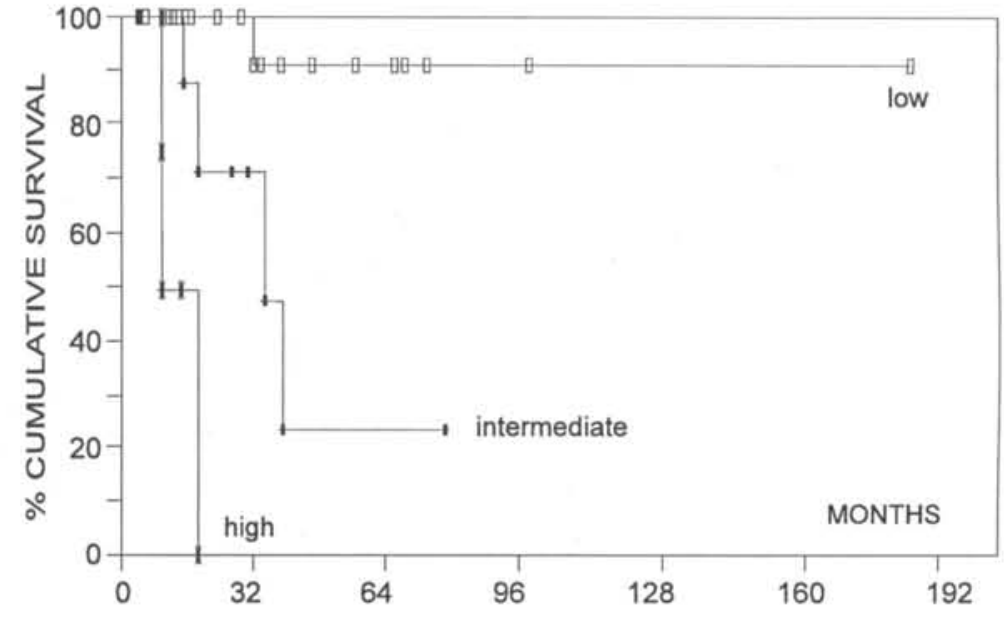

Figure 3 - Survival of patients with low, intermediate and high relative risk (RR), according to the equation derived from multivariate analysis $(p=0.00000)$. 
Table 2

Number, initials, sex, age (in years), race, spleen size (left rib edge, in centimeters), liver size (right rib edge, in centimeters), \% of hemoglobin ( $\mathrm{Hb}$ ), number of white blood cells (WBC) per cubic millimeter, platelets (per cubic millimeter) and erythroblasts (Erythro) in the peripheral blood of the $\mathbf{4 5}$ patients.

\begin{tabular}{|c|c|c|c|c|c|c|c|c|c|c|}
\hline Number & Initials & Sex & Age & Race & Spleen & Liver & $\mathrm{Hb}$. & WBC & Platelets & Erythro \\
\hline 1 & ADA & $M$ & 36 & W & 20 & 0 & 12 & 25100 & 750000 & 0 \\
\hline 2 & AFN & $M$ & 36 & W & 25 & 5 & 6 & 3200 & 45000 & 14 \\
\hline 3 & AS & $M$ & 57 & W & 5 & 2 & 6 & 140000 & 126000 & 0 \\
\hline 4 & AMMF & $M$ & 17 & B & 30 & 0 & 7 & 36000 & 200000 & 0 \\
\hline 5 & AAA & $\mathrm{F}$ & 25 & B & 12 & 0 & 10 & 170000 & 350000 & 0 \\
\hline 6 & BAA & $\mathrm{F}$ & 15 & B & 7 & 2 & 7 & 240000 & 360000 & 0 \\
\hline 7 & CMMP & $\mathrm{F}$ & 72 & W & 7 & 0 & 10 & 77300 & 332000 & 0 \\
\hline 8 & CSM & $\mathrm{F}$ & 38 & B & 25 & 2 & 11 & 96000 & 380000 & 0 \\
\hline 9 & CVS & $M$ & 56 & B & 15 & 1 & 13 & 140000 & 450000 & 8 \\
\hline 10 & $\mathrm{CL}$ & $F$ & 16 & B & 15 & 2 & 9 & 502000 & 730000 & 0 \\
\hline 11 & EV & $M$ & 57 & W & 6 & 0 & 12 & 141000 & 350000 & 0 \\
\hline 12 & EDN & $M$ & 62 & w & 8 & 0 & 11 & 68000 & 150000 & 0 \\
\hline 13 & ESC & $\mathrm{F}$ & 42 & W & 8 & 3 & 12 & 126800 & 300000 & 3 \\
\hline 14 & ERC & $\mathrm{F}$ & 64 & W & 25 & 0 & 6 & 700000 & 200000 & 4 \\
\hline 15 & FLJ & $M$ & 33 & B & 0 & 1 & 9 & 214000 & 160000 & 0 \\
\hline 16 & FBL & $\mathrm{F}$ & 33 & W & 20 & 0 & 11 & 400000 & 240000 & 1 \\
\hline 17 & $\mathrm{GC}$ & M & 64 & W & 7 & 5 & 7 & 37000 & 140000 & 3 \\
\hline 18 & HMA & $\mathrm{F}$ & 53 & W & 20 & 3 & 11 & 150000 & 210000 & 3 \\
\hline 19 & JAS & $M$ & 39 & B & 15 & 0 & 12 & 84000 & IGN & 0 \\
\hline 20 & $J M G$ & $M$ & 30 & W & 10 & 2 & 12 & 156000 & 400000 & 0 \\
\hline 21 & JNPF & $M$ & 62 & w & 15 & 3 & 9 & 176000 & 600000 & 0 \\
\hline 22 & JDJT & $M$ & 27 & w & 20 & 0 & 7 & 560000 & 150000 & 0 \\
\hline 23 & LE & $M$ & 61 & w & 20 & 0 & 10 & 190000 & 260000 & 0 \\
\hline 24 & LAS & M & 36 & W & 20 & 4 & IGN & IGN & IGN & 0 \\
\hline 25 & LCNF & $M$ & 31 & B & 10 & 2 & 13 & 122000 & 260000 & 1 \\
\hline 26 & LRP & $M$ & 25 & w & 16 & 4 & 11 & 80000 & 470000 & 1 \\
\hline 27 & MSA & $M$ & 27 & W & 23 & 4 & 7 & 280000 & 200000 & 0 \\
\hline 28 & MRC & $M$ & 21 & W & 18 & 4 & 9 & 192000 & 500000 & 0 \\
\hline 29 & MACA & $\mathrm{F}$ & 46 & B & 5 & 4 & 11 & 13500 & 1000000 & 2 \\
\hline 30 & MLNH & $\mathrm{F}$ & 58 & w & 10 & 0 & 11 & 180900 & 350000 & 0 \\
\hline 31 & MHS & $\mathrm{F}$ & 51 & w & 3 & 3 & 12 & 48000 & 420000 & 0 \\
\hline 32 & MCS & $M$ & 26 & B & 6 & 0 & 8 & 73000 & 260000 & 0 \\
\hline 33 & NP & $\mathrm{F}$ & 70 & w & 1 & 0 & 11 & 15500 & 282000 & 0 \\
\hline 34 & OT & $\mathrm{F}$ & 58 & w & 10 & 3 & 11 & 15400 & 350000 & 0 \\
\hline 35 & PBB & $M$ & 62 & W & 25 & 2 & 11 & 132900 & 777000 & 0 \\
\hline 36 & PS & $\mathrm{F}$ & 69 & W & 12 & 4 & 9 & 276000 & 595000 & 0 \\
\hline 37 & RTY & $M$ & 30 & A & IGN & IGN & IGN & IGN & IGN & 0 \\
\hline 38 & STM & $\mathrm{F}$ & 32 & B & 16 & 6 & 9 & 205800 & 450000 & 0 \\
\hline 39 & YM & $M$ & 77 & A & 4 & 4 & 11 & 58300 & 110000 & 0 \\
\hline 40 & MSS & $\mathrm{F}$ & 65 & B & 20 & 0 & 10 & 142000 & 160000 & 6 \\
\hline 41 & MCC & $\mathrm{F}$ & 42 & B & 0 & 0 & 12 & 80000 & 240000 & 0 \\
\hline 42 & LGNS & M & 27 & B & 10 & 0 & 10 & 348000 & 190000 & 0 \\
\hline 43 & JFS & $\mathrm{F}$ & 64 & B & IGN & 12 & 16 & 100000 & 450000 & 0 \\
\hline 44 & EGFR & $\mathrm{F}$ & 34 & B & IGN & IGN & IGN & IGN & IGN & 0 \\
\hline 45 & JFC & $M$ & 47 & B & 30 & 8 & 7 & 700000 & 550000 & 0 \\
\hline
\end{tabular}

Legend: $\mathrm{M}=$ Male; $\mathrm{F}=$ Female $; \mathrm{W}=$ White; $\mathrm{B}=$ Black; $\mathrm{A}=\mathrm{Asian} ; \mathrm{IGN}=$ ignored 
Table 3

Patient number (N), \% of blasts (BLAST), promyelocyte (PM) and myelocyte (M) in the peripheral blood, \% of blasts in the bone marrow (BMB), \% of eosinophils (EOSI) and basophils (BASO) in the peripheral blood, bone marrow M/E ratio (M/E), overall survival (OS) in months, post blast crisis survival (PBCS) in months, bone marrow biopsy classification (BMC), degree of fibrosis in bone marrow (FIBRO), score (SCORE) and relative risk (RR)

\begin{tabular}{|c|c|c|c|c|c|c|c|c|c|c|c|c|c|}
\hline № & BLAST & $\mathrm{PM}$ & $M$ & $\mathrm{BMB}$ & EOSI & BASO & $M / E$ & OS & PBCS & $\mathrm{BMC}$ & FIBRO & SCORE & RR \\
\hline 1 & 0 & 0 & 0 & 7 & 2 & 0 & $I G N$ & 10 & NC & $I G N$ & IGN & II & 1,20 \\
\hline 2 & 0 & 3 & 0 & 5 & 27 & 4 & 10 & 27 & NC & $\mathrm{G}$ & + & II & 1,93 \\
\hline 3 & 2 & 1 & 3 & 5 & 6 & 7 & 13 & 36 & 4 & $\mathrm{G}+\mathrm{M}$ & ++ & II & 4,27 \\
\hline 4 & 4 & 5 & 15 & 3 & 1 & 8 & 75 & 31 & 10 & $\mathrm{G}+\mathrm{M}$ & +++ & II & 3,09 \\
\hline 5 & 2 & 0 & 29 & 0 & 0 & 0 & 8 & 13 & NC & $\mathrm{G}+\mathrm{M}$ & ++ & II & 0,15 \\
\hline 6 & 4 & 0 & 9 & 0 & 2 & 2 & 12 & 13 & NC & $\mathrm{G}$ & + & 1 & 0,26 \\
\hline 7 & 4 & 8 & 4 & 0 & 5 & 0 & IGN & 30 & $\mathrm{NC}$ & IGN & IGN & 1 & 0,15 \\
\hline 8 & 2 & 5 & 16 & 0 & 5 & 3 & IGN & 12 & $\mathrm{NC}$ & $\mathrm{G}+\mathrm{M}$ & ++ & 1 & 0,34 \\
\hline 9 & 2 & 2 & 20 & 0 & 3 & 4 & 5 & 16 & NC & $\mathrm{G}+\mathrm{M}$ & + & 1 & 0,44 \\
\hline 10 & 5 & 3 & 40 & 1 & 1 & 3 & IGN & 48 & NC & $\mathrm{G}$ & 0 & 1 & 0,46 \\
\hline 11 & 3 & 5 & 5 & 1 & 4 & 0 & 19 & 39 & NC & G & ++ & 1 & 0,21 \\
\hline 12 & 5 & 14 & 15 & 1 & 4 & 4 & 13 & 97 & NC & $\mathrm{G}+\mathrm{M}$ & + & 1 & 0,60 \\
\hline 13 & 14 & 29 & 30 & 5 & 7 & 0 & 11 & 59 & 3 & $\mathrm{G}$ & 0 & II & 0,67 \\
\hline 14 & 5 & 6 & 30 & IGN & 4 & 2 & IGN & 1 & NC & IGN & IGN & IGN & IGN \\
\hline 15 & 0 & 0 & 0 & 2 & 4 & 0 & 10 & 33 & 8 & IGN & IGN & II & 0,28 \\
\hline 16 & 1 & 8 & 12 & 0 & 3 & 7 & 9 & 24 & NC & $I G N$ & IGN & II & 0,98 \\
\hline 17 & 0 & 6 & 6 & $I G N$ & 0 & 0 & IGN & 48 & NC & $I G N$ & IGN & IGN & IGN \\
\hline 18 & 28 & 2 & 5 & 3 & 1 & 2 & 100 & 35 & NC & $\mathrm{G}+\mathrm{M}$ & +++ & 1 & 0,63 \\
\hline 19 & 8 & 1 & 2 & 12 & 7 & 3 & 11 & 24 & 4 & $\mathrm{G}$ & ++ & III & IGN \\
\hline 20 & 0 & 5 & 13 & 10 & 3 & 10 & 9 & 10 & 1 & $I G N$ & IGN & III & 41,00 \\
\hline 21 & 2 & 1 & 3 & 0 & 2 & 10 & 6 & 34 & 3 & $\mathrm{G}+\mathrm{M}$ & +++ & II & 2,10 \\
\hline 22 & 5 & 2 & 4 & 5 & 5 & 12 & 30 & 10 & 5 & $\mathrm{G}$ & ++ & II & 16,00 \\
\hline 23 & 0 & 0 & 0 & 1 & 22 & 4 & 50 & 13 & $\mathrm{NC}$ & $\mathrm{G}+\mathrm{M}$ & ++ & II & 0,60 \\
\hline 24 & IGN & IGN & IGN & IGN & IGN & IGN & IGN & 18 & 7 & IGN & IGN & IGN & IGN \\
\hline 25 & 0 & 0 & 0 & 1 & 0 & 1 & IGN & 11 & NC & G & + & II & 0,20 \\
\hline 26 & 0 & 0 & 6 & 0 & 8 & 10 & 32 & 16 & 4 & $\mathrm{G}+\mathrm{M}$ & + & III & 2,10 \\
\hline 27 & 0 & 25 & 3 & 0 & 21 & 26 & 55 & 14 & NC & $\mathrm{G}$ & + & III & 150,30 \\
\hline 28 & 0 & 0 & 2 & 1 & 7 & 0 & 23 & 15 & NC & $\mathrm{G}+\mathrm{M}$ & ++ & II & 0,20 \\
\hline 29 & 0 & 0 & 1 & 1 & 1 & 5 & IGN & 66 & NC & $\mathrm{G}+\mathrm{M}$ & +++ & 1 & 0,70 \\
\hline 30 & 0 & 4 & 6 & 1 & 0 & 0 & 39 & 35 & NC & $\mathrm{G}+\mathrm{M}$ & ++ & 1 & 0,20 \\
\hline 31 & 0 & 0 & 7 & 0 & 1 & 1 & 18 & 18 & NC & $I G N$ & IGN & 1 & 0,20 \\
\hline 32 & 2 & 0 & 5 & $I G N$ & 1 & 8 & IGN & 33 & 1 & $I G N$ & IGN & IGN & IGN \\
\hline 33 & 0 & 1 & 1 & 0 & 2 & 2 & $I G N$ & 73 & NC & $I G N$ & IGN & 1 & 0,20 \\
\hline 34 & 0 & 1 & 2 & 0 & 4 & 4 & 6 & 184 & NC & IGN & IGN & 1 & 0,44 \\
\hline 35 & 1 & 1 & 8 & 8 & 4 & 0 & 21 & 78 & NC & $\mathrm{G}+\mathrm{M}$ & ++ & 1 & 1,62 \\
\hline 36 & 4 & 2 & 3 & 2 & 8 & 6 & 20 & 19 & 1 & $\mathrm{G}$ & +++ & II & 1,36 \\
\hline 37 & IGN & IGN & IGN & $I G N$ & IGN & IGN & IGN & 42 & 1 & $I G N$ & IGN & IGN & IGN \\
\hline 38 & 4 & 10 & 9 & 5 & 11 & 11 & 31 & 17 & 6 & IGN & IGN & II & 12,38 \\
\hline 39 & 2 & 0 & 3 & 3 & 0 & 2 & 48 & 68 & NC & $\mathrm{G}$ & ++ & 1 & 0,63 \\
\hline 40 & 0 & 0 & 1 & 2 & 4 & 0 & 24 & 7 & NC & $\mathrm{G}$ & ++ & 1 & 0,28 \\
\hline 41 & 0 & 0 & 2 & 1 & 3 & 1 & 22 & 5 & NC & $I G N$ & IGN & 1 & 0,27 \\
\hline 42 & 0 & 5 & 14 & 6 & 4 & 1 & IGN & 5 & NC & $\mathrm{G}+\mathrm{M}$ & +++ & II & 1,17 \\
\hline 43 & 0 & 0 & 0 & 0 & 2 & 0 & 17 & 7 & NC & $\mathrm{G}+\mathrm{M}$ & +++ & 1 & 0,15 \\
\hline 44 & IGN & 0 & 0 & 7 & IGN & IGN & 36 & 64 & 10 & $I G N$ & IGN & IGN & IGN \\
\hline 45 & 0 & 0 & 0 & 4 & 4 & 14 & 10 & 9 & NC & $I G N$ & IGN & II & 20,31 \\
\hline
\end{tabular}

Legend: $\mathrm{NC}=$ No blastic crisis; $I \mathrm{GN}=$ Ignored 
a Ph1-positive population during the first year after diagnosis, and 23-28 percent per year during the third to the fifth year. In another study ${ }^{20}$, the rates were 5 percent during the first year after diagnosis, 12 percent during the second and 22,5 percent per year during the next eight years. Our data is compatible: 5.1 percent in the first year, 18.1 percent in the second, 23.1 percent in the third, 18.2 percent in the fourth, and a "plateau" after the fifth year. The staging system proposed here permits our population to be divided into three distinct groups: low, intermediate and high risk, with significant differences between mean survivals and death rates. Along the same line, the multivariate analysis permitted the population to be divided into 3 groups (low, intermediate and high risk groups) with significant difference in survival ( $\mathrm{p}=$ 0.00000 ) which we believe is more accurate than the proposed staging system, which provides an equation that only includes prognostic factors with primary importance.

\section{CONCLUSIONS}

We conclude that there is the possibility of identifying some high-risk factors in a CML population with the aim of recognizing patients at a high risk of acceleration so as to schedule bone marrow transplantation as soon as possible. Further studies should be carried out in order to identify other poor prognostic factors in the CML Brazilian population, and to investigate the reason for high death rates among younger patients.

\section{Resumo}

Avaliamos o valor prognóstico de diferentes fatores, ao diagnóstico, em 45 pacientes com LMC Ph1-positivos, A sobrevida mediana foi de 48 meses. A análise univariada identificou 5 fatores associados a pior prognóstico (sexo masculino, idade inferior a 45 anos, blastos na medula óssea maior ou igual a 10 percent, basófilos no sangue periférico maior ou igual a 6 percent e eosinófilos no sangue periférico maior ou igual a 6 percent), originando um sistema de estadiamento: estágio 1 com zero ou um fator e sobrevida de 100 percent em dois anos; estágio II com dois ou três fatores e sobrevida de 72,2 percent em dois anos; estágio III com 4 ou 5 fatores e sobrevida de 0 percent em dois anos $(p=0.00016)$. A análise multivariada demonstrou que a basofilia no sangue periférico e os blastos na medula óssea foram os fatores que melhor se correlacionaram com o tempo de sobrevida. Concluimos que a combinaçóo de fatores presentes no diagnóstico permite a identificaçāo de diferentes grupos de risco na LMC, podendo ser útil na determinaçóo do prognóstico e na abordagem terapêutica.

\section{REFERENCES}

1. Alimena G, Morra E, Lazzarino M, et al. Interferon alfa$2 \mathrm{~b}$ as therapy for patients with $\mathrm{Ph} 1$-positive chronic myelogenous leukemia. Eur J Haematol 1990; 45(52):25-8.

2. Cervantes F, Rozman C. A multivariate analysis of prognostic factors in chronic myeloid leukemia. Blood 1982;60:1298-1304.

3. Champlin RE, Golde DW. Chronic myeloid leukemia: recent advances. Blood 1985;65(5):1039-1047.
4. Cox RD. Regression models and life-tables. J R Stat Soc B 1982;34:187-202.

5. Ezdinli EZ, Sokal JE, Crosswhite BS, Sandberg AA. Philadelphia-chromosome-positive and -negative chronic myelocytic leukemia. Ann Int Med 1970; 7(2):175-182.

6. Fitzgerald PH, Beard EJ, Morris CM, Heaton DC, Reeve $\mathrm{AE}$. Ph-negative chronic myeloid leukemia. Br J Haematol 1987;66:311-4.

7. Frisch B, Bartl R, Burkhardt R, Jager K, Mahl G, Kettner G. Classification of myeloproliferative disorders by bone marrow histology. Biblthca Haemat 1984;50:57-80. 
8. Gomez A, Sokal JE, Walsh D. Prognostic features at diagnosis of chronic myelocytic leukemia. Cancer 1981;47:2470-7.

9. Janossy G, Woodruff RK, Pippard MJ, et al. Relation of lymphoid phenotype and response to chemotherapy incorporating vincristine-prednisolone in the acute phase of CML Phl-positive leukemia. Cancer 1989;43:426-434.

10. Jootar S, Ongphiphaphadhanakul B, Chuncharunee S, Atichartakarn V. Multivariate analysis of prognostic factors in chronic myeloid leukemia. J Med Assoc Thai 1990;73(12):662-9.

11. Kantarjian MH, Smith TL, McCredie KB, et al. Chronic myelogenous leukemia; a multivariate analysis of the associations of patient characteristics and therapy with survival. Blood 1985;66(6):1326-1335.

12. Kaplan GL, Meier P. Nonparametric estimation from incomplete observations. J Am Stat Assoc 1958; 53:457481.

13. Lee ET. Statistical methods for survival data analysis. Lifetime Learning Publication. Belmont:Publication Lifetime Learning, 1980:551.
14. Rowley JD. The Philadelphia chromosome translocation: a paradigm for understanding leukemia. Cancer 1990;65(10):2178-2184.

15. Silver RT. Chronic myeloid leukemia: a perspective of the clinical and biologic issues of the chronic phase. Haematol/ Oncol. Clin N Am 1990;4(2):319-335.

16. Sjogren U, Brandt L, Mieltman F. Relation between life expectancy and composition of the bone marrow at diagnosis of chronic myeloid leukemia. Scand J Haematol 1974;12:369-373.

17. Sokal JE, Baccarani M, Tura S, et al. Prognostic discrimination among younger patients with chronic granulocytic leukemia: relevance of bone marrow transplantation. Blood 1985;66(6):1352-7.

18. Sokal JE, Cox EB, Baccarani M, et al. and the Italian Cooperative CML Study Group. Prognostic discrimination in "good risk" chronic granulocytic leukemia. Blood 1984;63(4):789-799.

19. Theologides A. Unfavorable signs in patients with chronic myeloid leukemia. Ann Int Med 76:95-9.

20. Tura S, Baccarani M, Corbelli, G and the Italian Cooperative Study Group On Chronic Myeloid Leukemia. Staging of chronic myeloid leukemia. Br J Haematol 1981;47:105-119. 